\title{
Results on Inclusive Diffraction from the ZEUS Experiment by the $M_{X}$-Method
}

\author{
Bernd Löhr, DESY \\ on behalf of the ZEUS Collaboration
}

\begin{abstract}
Diffractive deep inelastic scattering, $e p \rightarrow e^{\prime} \gamma^{*} p \rightarrow e^{\prime} X N$, has been studied at HERA with the ZEUS detector using an integrated luminosity of $52.4 \mathrm{pb}^{-1}$. The diffractive contribution to deep inelastic scattering has been determined with the $M_{X}$-method. The measurement covers a wide range in the $\gamma^{*} p$ c.m. energy $W(45-220 \mathrm{GeV})$, photon virtuality $Q^{2}\left(25-320 \mathrm{GeV}^{2}\right)$ and mass $M_{X}(1.2-30 \mathrm{GeV})$. We present preliminary results on the diffractive structure function, $x_{I P} F_{2}^{D(3)}$. For comparison, results from our previous measurement at $Q^{2}=2.7-55 \mathrm{GeV}^{2}$ are also included.
\end{abstract}

\section{The $M_{X}$-Method}

$M_{X}$ is defined as the mass from all measured particles in the detector, except the scattered electron. The shape of the $M_{X}$-distribution is different for nondiffractive and diffractive events. Nondiffractive events lead to a rapidity-plateau distribution for the produced particles. Particle emission is a statistical process which may lead to a rapidity gap. The probability to find a rapidity gap $\Delta y$ is given by Poisson statistics, $P(0)=e^{-\lambda \Delta y}$ where $\lambda$ is the height of the plateau. This leads to the following mass distribution for nondiffractive events:

$$
\frac{d N_{\text {nondiff }}}{d \ln M_{X}^{2}}=c \cdot e^{b \cdot \ln M_{X}^{2}} .
$$

Diffractive events show a different $M_{X}$-distribution. For not too low and not too high values of $M_{X}$ one finds experimentally [2]

$$
\frac{d N_{\text {diff }}}{d M_{X}^{2}} \propto \frac{1}{M_{X}^{2}} \text { from which follows } \frac{d N_{\text {diff }}}{d \ln M_{X}^{2}} \approx \text { const. }
$$

This can also be derived from a Triple Regge Model [3]. For the sum of nondiffractive and diffractive events one gets :

$$
\frac{d N}{d \ln M_{X}^{2}}=D+c \cdot e^{b \cdot \ln M_{X}^{2}}
$$

Figure 1 shows a measured $\ln M_{X}^{2}$-distribution. It is compared to the properly normalized distribution of the sum of MC-simulated nondiffractive and diffractive (hatched) events. Over the range $\ln M_{X}^{2} \leq \ln W^{2}-\eta_{0}$, which is indicated by two vertical lines, the above formula with $D=$ const. is fitted to the measured distribution with $\eta_{0}$ taken from data. This gives a very good fit for the nondiffractive contribution $c \cdot e^{b \cdot \ln M_{X}^{2}}$. The fitted nondiffractive contribution is subtracted statistically from the data for each $\ln M_{X}^{2}$-bin to obtain the number of diffractive events in that bin. In the analysis only bins are used in which the diffractive part is at least $50 \%$. The diffractive events selected by the $M_{X}$-method contain contributions from proton-dissociative events. 
This contribution was estimated in the following way. Events were selected from a kinematical region which is dominated by proton-dissociative events and show energy deposited in the detector from the proton-dissociative system $M_{N}$. A MCsimulation of proton dissociation has been tuned to describe these events. As a result, the number of proton-dissociative events with a generated mass $N_{N} \geq 2.3 \mathrm{GeV}$ can be well described by the MC-simulation. For each kinematical $\left(Q^{2}, W, M_{X}\right)$-bin the corresponding number of MC-simulated events from proton-dissociation with masses $M_{N} \geq 2.3 \mathrm{GeV}$ has been subtracted statistically from the diffractive data. The ZEUS inclusive diffractive data selected with the $M_{X}$-method therefore contain contributions from proton-dissociative events with $M_{N}<2.3 \mathrm{GeV}$.

\section{The Diffractive Structure Function}

The inclusive diffractive cross section in DIS can be expressed in terms the diffractive structure function in the same way as the inclusive DIS cross section is expressed by the DIS structure function. The inclusive diffractive process in DIS is described by the differential cross section:

$$
\begin{aligned}
& \frac{d^{3} \sigma_{\gamma^{*} p \rightarrow X N}^{\text {diff }}}{d Q^{2} d \beta d x_{I P}}=\frac{2 \pi \alpha_{e m}^{2}}{\beta Q^{2}}\left[1+(1-y)^{2}\right] \cdot F_{2}^{D(3)}\left(\beta, x_{I P}, Q^{2}\right) \\
& \text { with } \quad x_{I P}=\frac{Q^{2}+M_{X}^{2}}{Q^{2}+W^{2}} \text { and } \beta=\frac{x}{x_{I P}}=\frac{Q^{2}}{Q^{2}+M_{X}^{2}}
\end{aligned}
$$

The contribution from the longitudinal structure function $F_{L}^{D(3)}$ is small in the kinematic region of the presented analysis and is neglected.

If $F_{2}^{D(3)}\left(\beta, x_{I P}, Q^{2}\right)$ is interpreted in terms of quark densities it specifies the probability to find in a proton, which undergoes a diffractive interaction, a quark carrying the fraction $x=\beta x_{I P}$ of the proton momentum.

\section{Results from the $M_{X}$-Method}

In an earlier publication, ZEUS presented results [3] on inclusive diffraction obtained with the $M_{X}$-method which covered the range $2.7 \mathrm{GeV}^{2} \leq Q^{2} \leq 55 \mathrm{GeV}^{2}$. In this contribution new preliminary results are presented for higher values for $Q^{2}$, namely $25 \mathrm{GeV}^{2} \leq Q^{2} \leq$ $320 \mathrm{GeV}^{2}$. The two sets of results are called FPC I and FPC II in what follows.

In the kinematical bins $Q^{2}=25 \mathrm{GeV}^{2}$ and $Q^{2}=55 \mathrm{GeV}^{2}$, where results from both data sets exist, the cross sections agree within the errors. The structure function $x_{I P} F_{2}^{D(3)}$ as 
function of $x_{I P}$ obtained with the $M_{X}$-method is displayed in Figure 2 for the two datasets FPCI and FPC II. In all kinematic bins, a pronounced rise of $x_{I P} F_{2}^{D(3)}$ with decreasing $x_{I P}$ is visible. Also shown is the result of a simultaneous fit to all the data using a modified BEKWmodel [4] in a slightly modified version [3]. The BEKW-model is a dipole model which parametrizes $x_{I P} F_{2}^{D(3)}$ in terms contributions from: transverse photon $\rightarrow q \bar{q}$, longitudinal photon $\rightarrow q \bar{q}$, and transverse photon $\rightarrow q \bar{q} g:$

$$
x_{I P} F_{2}^{D(3)}\left(\beta, x_{I P}, Q^{2}\right)=c_{T} \cdot F_{q \bar{q}}^{T}+c_{L} \cdot F_{q \bar{q}}^{L}+c_{g} \cdot F_{q \bar{q} g}^{T} .
$$

The modified BEKW parametrization has five free parameters which have to be determined by a fit to the data: the normalizations of the three contributions, $c_{T}, c_{L}, c_{g}$, a coefficient, $n^{T, g}$, which determines the $x_{I P}$-dependence of the transverse photon and of the $q \bar{q} g$-contribution, and a coefficient, $\gamma$, which determines the $\beta$-dependence of the $q \bar{q} g$-contribution. A fit of these five parameters to the combined FPC I + II data yield $\chi^{2} / n_{D}=0.82$ taking into account the full errors. The full line in Figure 2 shows the result of the fit. Also shown are the individual contributions. For $0.2<\beta<0.9$ the $(q \bar{q})_{T}$ contribution dominates. The gluon emission term $q \bar{q} g$ gives the largest contribution for $\beta<0.15$. The longitudinal term $(q \bar{q})_{L}$ dominates for $\beta>0.9$.

\section{References}

[1] Slides: http: //indico. cern. ch/contributionDisplay . py? contribId=60\&sessionId=7\& conf Id=9499

[2] K. Goulianos, Phys. Reports 101 (1983) 169; CDF Coll., F.Abe et al., Phys. Rev. D50 (1994) 5535.

[3] ZEUS Coll., S. Chekanov et al., Nucl. Phys. B 713 (2005) 3.

[4] J. Bartels et al., Eur. Phys. J. C7 (1999) 443. 

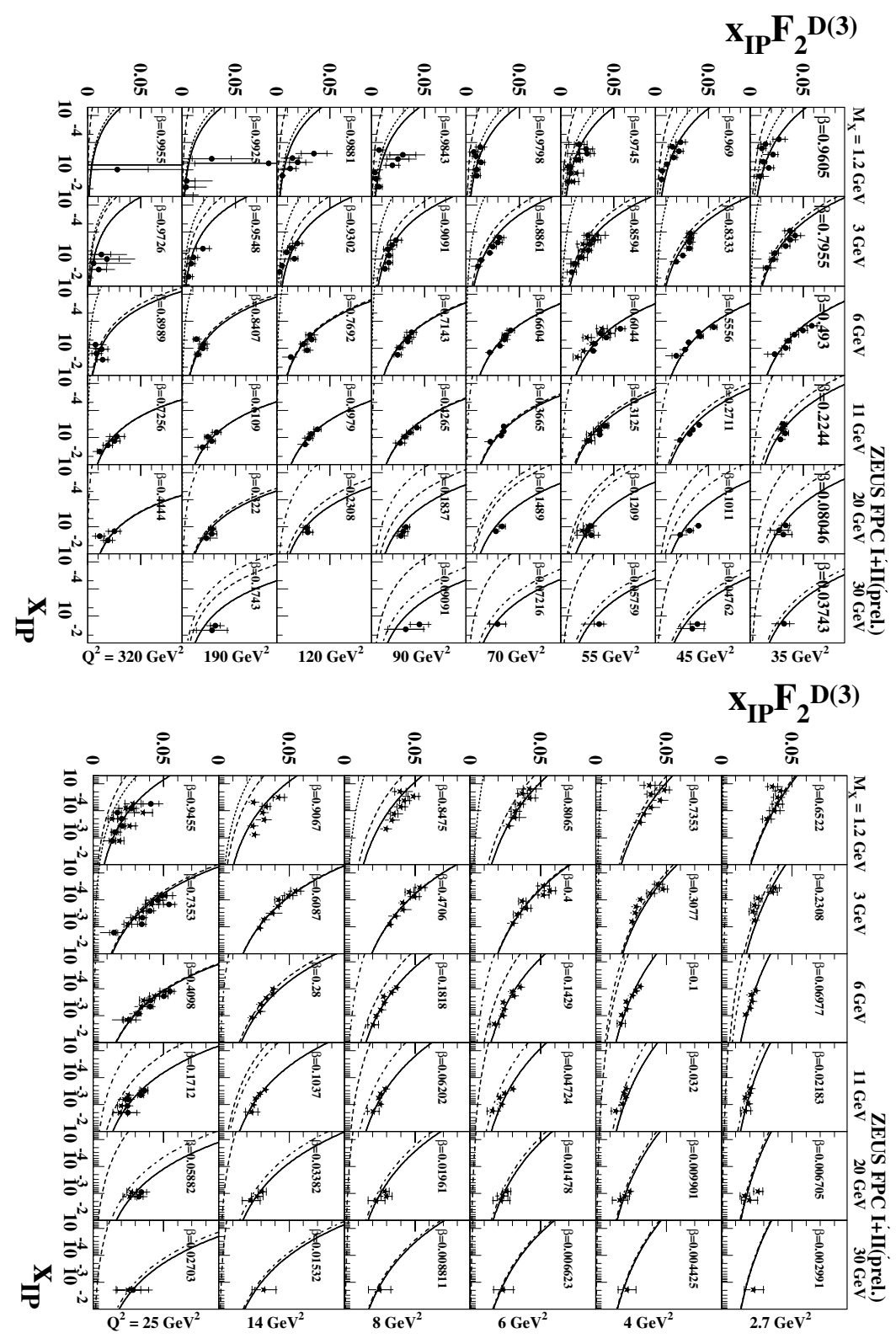

Figure 2: The diffractive structure function of the proton multiplied by $x_{I P}, x_{I P} F_{2}^{D(3)}$, for $\gamma^{*} p \rightarrow X N, M_{N}<2.3 \mathrm{GeV}$ as a function of $x_{I P}$ for different regions of $\beta$ : FPC I and FPC II results are presented. The inner error bars show the statistical uncertainties and the full bars the statistical and systematic uncertainties added in quadrature. The curves show the results of the BEKW fit for the contributions from $(q \bar{q})$ for transverse (dashed) and longitudinal photons (dotted) and for the $(q \bar{q} g)$ contribution (dashed-dotted) together with the sum of all contributions (solid). 